\title{
Motiviert begonnen, erfolgreich beendet?
}

\section{Prädiktive Kraft der Studienwahlmotivation für Noten im Lehramtsstudium}

\author{
Caroline Theurer ${ }^{1} @$, Victoria Bleck ${ }^{1}$, Katharina Deistler ${ }^{2}$ und Frank Lipowsky ${ }^{1}$ \\ 1Fachgebiet Empirische Schul- und Unterrichtsforschung, Institut für Erziehungswissenschaft, Universität Kassel, Deutschland \\ ${ }^{2}$ PFH Private Hochschule Göttingen, Deutschland
}

\begin{abstract}
Zusammenfassung: Die Bedeutung der Studienwahlmotivation für den Studien- und Berufserfolg von Lehrkräften wird vielfach betont, wurde bislang jedoch nicht hinreichend untersucht. So ist zwar bekannt, dass die Studienwahlmotivation mit leistungsrelevanten Merkmalen wie der Nutzung von Lernstrategien in Verbindung steht, unklar ist jedoch, inwiefern die Studienwahlmotivation darüber hinaus auch Leistungsmaße beeinflusst. Im vorliegenden Beitrag wird die prognostische Validität der Studienwahlmotivation auf zwei Leistungsmaße geprüft: a) die selbstberichtete Abschlussnote und b) die kumulierten Noten im Studienverlauf. Dabei werden Wirkungen von Primärfaktoren (Pädagogisches Interesse, Fachliches Interesse, Fähigkeitsüberzeugung, Soziale Einflüsse, Geringe Schwierigkeit des Studiums, Nützlichkeit) und Sekundärfaktoren (intrinsische und extrinsische Motivation) der mit dem FEMOLA erfassten Studienwahlmotivation untersucht. Dafür wird eine Stichprobe von Lehramtsstudierenden der Universität Kassel herangezogen (Projekt STUVE; N = 464). Lineare Regressionsanalysen unter Kontrolle des Geschlechts, der studierten Schulstufe und der Abiturnote zeigen, dass sowohl die intrinsische als auch die extrinsische Studienwahlmotivation schwach mit der selbstberichteten Abschlussnote sowie den kumulierten Noten im Studienverlauf zusammenhängen. Je höher die intrinsische Studienwahlmotivation und je niedriger die extrinsische Studienwahlmotivation, desto besser fallen jeweils die Noten der Lehramtsstudierenden aus. Betrachtet man zusätzlich die Wirkungen der Primärfaktoren der Studienwahlmotivation, ergeben sich für die beiden abhängigen Variablen teils unterschiedliche Zusammenhangsmuster: Während die selbstberichtete Abschlussnote durch keinen der Primärfaktoren vorhergesagt werden kann, korrespondieren die kumulierten Noten im Studium mit den Faktoren Fähigkeitsüberzeugung und einer antizipierten Geringen Schwierigkeit des Studiums. Die Ergebnisse werden kritisch diskutiert und unter Berücksichtigung der Limitationen der Studie in ihrer Bedeutung für die Lehrerbildungsforschung interpretiert.
\end{abstract}

Schlüsselwörter: Noten, Studienwahlmotivation, FEMOLA, Lehramtsstudierende, Lehrerbildung

Does motivation in the beginning equal success in the end? The predictive power of career choice motivation for grades during teacher education.

Abstract: There are profound theoretical arguments on the relevance of career choice motivation for teachers' academic and career success. Empirical studies are scarce but show that career choice motivation is related to performance-related features such as the use of learning strategies. However, it is unclear to what extent career choice motivation also influences the grades until the end of teacher education. Therefore, this paper relates first-order (intrinsic and extrinsic motivation) and second-order factors of career choice motivation (educational interest, subject-specific interest, utility, ability beliefs, low difficulty of the study, and social influences) as assessed by the FEMOLA-instrument to two achievement measures: a) self-reported final grade after finishing their studies and b) the students' cumulated performances during their studies, excluding the performance in their final exams. The sample consists of 464 teacher students from the University of Kassel. Controlling for gender, studied school level and the grade of university entrance qualification, linear regression models show low, but significant relations between intrinsic as well as extrinsic career choice motivation and both performance measures. The higher intrinsic and the lower extrinsic career choice motivation was, the better the performance. A closer look at first-order factors of career choice motivation reveals different patterns: Students' cumulated performance during their studies is related to the first-order factors ability beliefs and low difficulty of the study. The self-reported final grade, however, cannot be predicted by any of the first-order factors. The results are discussed critically when related to teacher education research. Considering the limitations of the study, further research perspectives are pointed out.

Keywords: Grades, pre-service teachers, career choice motivation, FEMOLA, teacher education 


\section{Einleitung}

Professionelles Lehrerhandeln wird theoretisch-konzeptionell durch Professionswissen, Überzeugungen, selbstregulative Fähigkeiten und motivationale Orientierungen determiniert (Baumert \& Kunter, 2006). Motivationale Orientierungen umfassen u.a. die Zielorientierungen von Lehrkräften, ihr Interesse und ihren Enthusiasmus, aber auch ihre Lehrerselbstwirksamkeit (zsf. Kunter, 2014). Ein weites Feld innerhalb der Forschung zur Lehrermotivation widmet sich zudem den Gründen für die Wahl des Lehramtsstudiums bzw. des Lehrberufs (Biermann et al., 2019; König, Drahmann \& Rothland, 2018; Kunter, 2014; Rothland, 2014): der Studien- bzw. Berufswahlmotivation. Dabei wird davon ausgegangen, dass die Studienwahlmotivation Einfluss auf die Adaptivität und Funktionalität des Studierverhaltens nimmt (Kunter, 2014). Theoretisch ist es plausibel, dass unterschiedlich gelagerte motivationale Voraussetzungen von Lehramtsstudierenden die Wahrnehmung und Nutzung von Lerngelegenheiten im Rahmen des Studiums beeinflussen (Kunter, Kleickmann, Klusmann \& Richter, 2011) und in unterschiedlichem Maße lernrelevante Verhaltensweisen gezeigt werden (König \& Rothland, 2012; Rothland, 2014). Dies sollte sich mittelfristig auch in unterschiedlichem Lernerfolg der Studierenden sowie langfristig in Unterschieden in den professionellen Kompetenzen der Lehrpersonen widerspiegeln.

Diese Annahmen zur Bedeutung der Studienwahlmotivation für Leistungsmaße im Studium wurden bislang jedoch kaum empirisch geprüft. Vorliegende Untersuchungen weisen häufig ein querschnittliches Design auf oder erfragen Motivation retrospektiv, was zu Verzerrungen führen kann (Kunter, 2014). Insgesamt lässt sich ein Mangel an längsschnittlichen Studien zur Wirkung der Studienwahlmotivation auf Leistungsmaße im Lehramtsstudium konstatieren (König \& Rothland, 2012; König et al., 2018; Rothland, 2014). Von diesem Forschungsdesiderat ausgehend widmet sich dieser Beitrag dem Einfluss der Studienwahlmotivation von Lehramtsstudierenden zu Studienbeginn auf die Leistungen bis zum Studienende. Operationalisiert werden die Studienleistungen über a) kumulierte Noten im Studienverlauf sowie b) über die selbstberichtete Note im Ersten Staatsexamen. Gemäß der häufigen Differenzierung zwischen intrinsischer und extrinsischer Studienwahlmotivation (z.B. Brookhart \& Freeman, 1992; Fray \& Gore, 2018; Pohlmann \& Möller, 2010; Richardson \& Watt, 2014) wird in diesem Beitrag einerseits der Effekt der intrinsischen und extrinsischen Motivation auf die beiden Kriterien untersucht. Zum Zwecke einer weiteren Differenzierung wird andererseits auch die Vorhersagerelevanz der Primärfaktoren für die beiden Kriterien geprüft.

\section{Gründe für die Wahl des Lehramtsstudiums bzw. Lehrberufs}

Es lassen sich vielfältige Gründe für die Wahl des Lehrberufs differenzieren (zsf. Cramer, 2016a; Rothland, 2014): Darunter fallen der Wunsch, mit Kindern und Jugendlichen $\mathrm{zu}$ arbeiten, die gesellschaftliche Relevanz des Lehrberufs, eigene schulische Erfahrungen sowie pädagogisches oder fachliches Interesse. Aber auch Nützlichkeitsaspekte, wie die antizipierte Freizeit sowie der Wunsch, berufliche und private Belange verknüpfen zu können, bewegen Studierende zur Aufnahme eines Lehramtsstudiums. Die Forschung zeigt, dass bei der Wahl des Lehramtsstudiums bzw. Lehrberufs beziehungsorientierte Motive dominieren und (zukünftige) Lehrkräfte insgesamt eher intrinsisch motiviert sind (Brookhart \& Freeman, 1992; Cramer, 2016a; Fray \& Gore, 2018; Heinz, 2015; Richardson \& Watt, 2014).

Der Terminus intrinsische Motivation meint dabei das Ausführen einer Handlung um ihrer selbst willen, während extrinsische Motivation jene Aktivierung beschreibt, die dazu dient, positive Konsequenzen herbeizuführen oder negative zu vermeiden (z. B. Rheinberg \& Vollmeyer, 2012). Bezogen auf die Studien- und Berufswahl umfasst die intrinsische Motivation das fachliche sowie pädagogische Interesse und die berufsbezogene Fähigkeitsüberzeugung. Unter extrinsischer Studienwahlmotivation werden Aspekte der Nützlichkeit, eine erwartete geringe Schwierigkeit des Studiums sowie soziale Einflüsse subsumiert (Pohlmann \& Möller, 2010). Wenngleich diese Unterschiedlichkeit in den Motivationsformen suggeriert, dass es sich bei intrinsischer und extrinsischer Motivation um gegensätzliche Pole handelt, können Individuen grundsätzlich in gleichem Maße intrinsisch wie extrinsisch motiviert sein (z. B. Deci \& Ryan, 1993).

\section{Bedeutung der Studienwahlmotivation für Studienleistungen}

Meist wird davon ausgegangen, dass die intrinsische Motivation im Vergleich zur extrinsischen Motivation günstiger für Anpassungs- und Lernleistungen ist, da sie Individuen eher dazu befähigen sollte, sich ausdauernd und konzentriert mit einem Gegenstand auseinanderzusetzen. Insbesondere in Bezug auf die Lernmotivation gilt sie als hohes pädagogisch-didaktisches Gut: Lernt ein Individuum in erster Linie aufgrund von Freude oder Interesse, dürften die effektivsten und nachhaltigsten Lernergebnisse erzielt werden (Köller, 1998; Paulick, 2011; Schunk, Meece \& Pintrich, 2014). Analog wird angenommen, dass sich die intrinsische Studien- und Berufswahlmotivation günstig auf die Bewährung in Studium und 
Beruf auswirkt (Mayr, 2009). In diesem Sinne erscheint es plausibel, dass eine überwiegend intrinsisch motivierte Studien- oder Berufswahl die Lernmotivation im Studium beeinflusst und dazu beiträgt, dass Lerninhalten mehr Bedeutung beigemessen wird und diese ausdauernder sowie tiefer verarbeitet werden, als wenn die Studien- oder Berufswahl vornehmlich extrinsisch motiviert ist (Rothland, 2014). Nicht zuletzt sollte das Vorliegen intrinsischer Studien- bzw. Berufswahlmotive dazu führen, dass eine höhere Passung von Inhalten des Studiums und individuellen Neigungen wahrgenommen wird (Holland, 1997), was sich günstig auf die Studienanpassung und damit auch auf die Leistungen im Studium auswirken dürfte.

Wendet man sich empirischen Befunden $\mathrm{zu}$, finden sich bislang kaum Studien, in welchen der Zusammenhang der Studienwahlmotivation von Lehramtsstudierenden mit den Studienabschlussnoten im Längsschnitt betrachtet wird. Metaanalysen liefern Hinweise auf Zusammenhänge zwischen intrinsischer Lernmotivation mit Leistungstests und Noten von Schülerinnen und Schülern sowie Studierenden $(r=.21$; Schiefele \& Schreyer, 1994) bzw. zwischen dem Interesse und schulischen Leistungen $(r=.30$; Schiefele, Krapp \& Schreyer, 1993). Dieser Befund lässt sich für den Zusammenhang von Studieninteresse und Studienleistung in einer Stichprobe von 92 Studierenden verschiedener Studiengänge im Längsschnitt über zwei Jahre replizieren (Schiefele, Wild \& Winteler, 1995).

Entsprechend der Fragestellung des Beitrags werden im Folgenden Ergebnisse von Untersuchungen mit Lehramtsstudierenden berichtet. In einigen Untersuchungen wurde der Einfluss der Studienwahlmotivation auf die Strategienutzung im Studium in den Blick genommen. Mayr (2009) untersuchte die Strategienutzung, indem er in einer der wenigen Längsschnittstudien die Wirkung der Studienwahlmotivation zu Studienbeginn auf die Lernstrategien im Studium überprüfte. Während intrinsische Studienwahlmotivation sich schwach positiv auf die Nutzung von Lernstrategien auswirkte, zeigt sich in dieser Studie für die extrinsische Studienwahlmotivation ein schwach negativer Einfluss. Auch die Ergebnisse der längsschnittlichen Untersuchung von Cramer (2012) deuten darauf hin, dass die intrinsische Studienwahlmotivation mit dem Lern- und Arbeitsverhalten im Studium in Zusammenhang steht. In der querschnittlichen Studie von Künsting und Lipowsky (2011) stellt neben der Gewissenhaftigkeit und der Lernzielorientierung zwar die intrinsische Studienwahlmotivation einen signifikanten Prädiktor der Nutzung von Lernstrategien dar, nicht jedoch die extrinsische Studienwahlmotivation. Mittels Profilanalysen konnten Streblow und Pohlmann (2017) nachweisen, dass sich stärker intrinsisch und stärker ex- trinsisch motivierte Studierende signifikant darin unterscheiden, wie sie Lernstrategien im Studium einsetzen: Erstere geben an, intensiver Elaborationsstrategien anzuwenden, um Wissen aufzubauen. Ähnliche Ergebnisse liefern auch Dörrenbacher-Ulrich, Biermann, Brünken und Perels (2019).

Was den Zusammenhang zwischen Berufswahlmotivation und Leistungsmaßen anbelangt, ermittelten König und Rothland (2013) zu Studienbeginn, dass im Querschnitt schwach positive Zusammenhänge des Wissens mit Faktoren intrinsischer Berufswahlmotivation bestehen $(r \approx .10)$. Zwischen den Faktoren extrinsischer Berufswahlmotivation (Verlegenheitslösung, Vereinbarkeit von Familie und Beruf) und dem pädagogischen Wissen bestehen hingegen keine signifikanten Zusammenhänge (König \& Rothland, 2013; s.a. König \& Rothland, 2012). Weiterführende längsschnittliche Analysen über den Zeitraum von einem Jahr zeigen entgegen der Erwartungen, dass der Faktor berufliche Sicherheit positiv mit dem Wissenszuwachs zusammenhängt, während die anderen Faktoren in keinem Zusammenhang mit dem Wissenszuwachs stehen (König \& Rothland, 2012).

Studien zum Zusammenhang von Studienwahlmotivation und erzielten Noten von Lehramtsstudierenden sind rar. Dörrenbacher-Ulrich et al. (2019) fanden keine Unterschiede in den Klausurleistungen zwischen unterschiedlich zur Studienwahl motivierten Profilen von Lehramtsstudierenden. Schwippert, Feld, Doll und Buchholtz (2013) untersuchten den Zusammenhang zwischen motivationalen und volitionalen Merkmalen Lehramtsstudierender einerseits und selbstberichteten Noten andererseits. Sie berichten u.a. Zusammenhänge zwischen fachlichem Studieninteresse und der fachwissenschaftlichen Studienleistung sowie zwischen fachdidaktischem Studieninteresse und der fachdidaktischen Studienleistung. Die berufsbezogene extrinsische Motivation hängt dagegen nicht mit den Studienleistungen zusammen. Nach den Ergebnissen von Mayr (2009) geht die intrinsische Studienwahlmotivation zu Studienbeginn mit einer besseren Note in der Diplomarbeit einher, während höhere Ausprägungen der extrinsischen Motivation mit einer schlechteren Note assoziiert sind. In die gleiche Richtung weisen Befunde von Brühwiler (2001), wonach Studierende des Primarstufenlehramts, die zu Beginn des Studiums angeben, wenig Freude an der Arbeit mit Kindern oder an der Tätigkeit zu haben, schlechtere Diplomnoten erzielten (Brühwiler, 2001). Zwar beziehen sich die beiden letztgenannten Studien nicht auf Lehramtsstudierende einer deutschen Universität, allerdings berichten König, Rothland, Darge, Lünnemann und Tachtsoglou (2013) von vergleichbaren Zusammenhangsmustern der Studienwahlmotivationsfaktoren in Deutschland, Österreich und der Schweiz (s. a. König et al., 2018), sodass zu- 
mindest von ähnlichen Mustern und Ausprägungen der Motivationsfaktoren in den deutschsprachigen Ländern ausgegangen werden kann.

Zusammenfassend legen die Befunde einen (schwachen) positiven Einfluss intrinsischer Faktoren der Studien- und Berufswahlmotivation auf Studienleistungen nahe. Für die Faktoren extrinsischer Studien- und Berufswahlmotivation zeichnet die Forschung eher ein heterogenes Bild: Teils sind Einflüsse auf Maße der Studienleistungen bzw. ihr naher Prädiktoren positiv (König \& Rothland, 2012), teils negativ (Beckmann, 2016; Mayr, 2009) oder nicht bedeutsam (König \& Rothland, 2013; Künsting \& Lipowsky, 2011; Schwippert et al., 2013). Gründe für die heterogene Befundlage könnten Unterschiede in der Operationalisierung der Kriterien, in den eingesetzten Instrumenten zur Erfassung der Motivation sowie in den Erhebungszeiträumen sein.

\section{Fragestellungen der Untersuchung}

Die Fülle an Untersuchungen zur Studien- und Berufswahlmotivation von Lehramtsstudierenden drückt deren Bedeutsamkeit für den Studienverlauf und -erfolg aus. Während die Ausprägung von Faktoren der Studienwahlmotivation häufig im Fokus der Forschung stand, wurde die prognostische Validität der Studien- bzw. Berufswahlmotivation für spätere Leistungen von Lehramtsstudierenden, vor allem gemessen in Form von Noten, seltener in den Blick genommen (Pohlmann \& Möller, 2010; Rothland, 2014). Daher beschäftigt sich der Beitrag mit der Frage, wie sich die Studienwahlmotivation, erfasst zu Studienbeginn, auf die Noten im Studium auswirkt. Sowohl die selbstberichtete Abschlussnote als auch die Noten im Studienverlauf sind als Indikator für die Leistungen aus verschiedenen Fachgebieten über mehrere Jahre zu verstehen und können als ein kumuliertes Maß für ein erfolgreiches Studium herangezogen werden. Dabei wird einerseits zwischen einem intrinsischen und einem extrinsischen Sekundärfaktor der Studienwahlmotivation sowie andererseits zwischen den Primärfaktoren nach Retelsdorf und Möller (2012) differenziert.

Forschungsfrage I: Hängt die intrinsische Studienwahlmotivation zu Studienbeginn mit der selbstberichteten Abschlussnote zusammen?

Forschungsfrage II: Hängt die extrinsische Studienwahlmotivation zu Studienbeginn mit der selbstberichteten Abschlussnote zusammen?

Forschungsfrage III: Hängt die intrinsische Studienwahlmotivation zu Studienbeginn mit den kumulierten Noten im Studienverlauf zusammen?
Forschungsfrage IV: Hängt die extrinsische Studienwahlmotivation zu Studienbeginn mit den kumulierten Noten im Studienverlauf zusammen?

Forschungsfrage V: In welchem Zusammenhang stehen die Faktoren der Studienwahlmotivation mit der selbstberichteten Abschlussnote?

Forschungsfrage VI: In welchem Zusammenhang stehen die Faktoren der Studienwahlmotivation mit den kumulierten Noten im Studienverlauf?

Aufgrund bisheriger Befunde wird ein schwacher positiver Zusammenhang zwischen der intrinsischen Studienwahlmotivation und einerseits den selbstberichteten Abschlussnoten (Hypothese 1) sowie andererseits den kumulierten Noten im Studienverlauf (Hypothese 2) postuliert. Für den Zusammenhang extrinsischer Studienwahlmotivation mit Leistungsmaßen kann aufgrund uneindeutiger empirischer Befunde und theoretisch unterschiedlicher Wirkungsmechanismen keine Erwartung über die Richtung des Zusammenhangs abgeleitet werden. Die Forschungsfragen II und IV werden daher explorativ bearbeitet.

Die Primärfaktoren fokussierend, kann auf Basis des Forschungsstands (Brühwiler, 2001; Dörrenbacher-Ulrich et al., 2019; Streblow \& Pohlmann, 2017) erwartet werden, dass sich schwache positive Zusammenhänge zwischen dem Pädagogischen Interesse und der selbstberichteten Abschlussnote (Hypothese 3) sowie der Fähigkeitsüberzeugung und der selbstberichteten Abschlussnote (Hypothese 4) ergeben. Zusätzlich lässt sich mit den Ergebnissen von Schwippert und Kollegen (2013) herleiten, dass diese Zusammenhangsstruktur auch für Fachliches Interesse gilt (Hypothese 5). Die beschriebenen Zusammenhänge des Pädagogischen Interesses, der Fähigkeitsüberzeugung sowie des Fachlichen Interesses werden analog mit den kumulierten Noten im Studienverlauf als abhängiger Variable angenommen (Hypothesen 6 bis 8). Für die Faktoren extrinsischer Studienwahlmotivation sind Erwartungen weit weniger eindeutig ableitbar, da differenzielle Wirkmechanismen denkbar sind. Es werden daher keine Hypothesen formuliert.

\section{Methode}

Für den vorliegenden Beitrag werden Daten aus dem Projekt STUVE (Studienverlauf und -erfolg Kasseler Lehramtsstudierender; Billich-Knapp, Künsting \& Lipowsky, 2012; Künsting \& Lipowsky, 2011) verwendet. Im Rahmen des Projekts, das an der Universität Kassel durchgeführt wurde, wurden Studierende für das Lehramt an Grundschulen, an Haupt- und Realschulen sowie an Gymnasien aus fünf Kohorten (Studienbeginn Wintersemester 2007/ 
Tabelle 1. Dropout-Analyse

\begin{tabular}{|c|c|c|c|c|c|c|c|c|}
\hline & \multicolumn{2}{|c|}{$\begin{array}{c}\text { Substichprobe mit } \\
\text { Noten }(N=464)\end{array}$} & \multicolumn{2}{|c|}{$\begin{array}{l}\text { Substichprobe ohne } \\
\text { Noten }(N=1,505)\end{array}$} & \multicolumn{4}{|c|}{ Prüfstatistiken } \\
\hline & $N$ & $\%$ & $N$ & $\%$ & $x^{2}$ & & $(d f)$ & $p$ \\
\hline Geschlecht: weiblich & 384 & 82.9 & 1,017 & 68.3 & 37.14 & & (1) & $<.001$ \\
\hline Lehramt GS & 175 & 38.0 & 367 & 24.8 & 31.19 & & (2) & $<.001$ \\
\hline \multirow[t]{2}{*}{ Lehramt HRS } & 111 & 24.1 & 390 & 26.4 & & & & \\
\hline & $M$ & $S D$ & $M$ & $S D$ & $t$ & $(d f)$ & $p$ & $d$ \\
\hline Alter & 21.09 & 3.84 & 21.38 & 3.63 & 1.50 & $(1,945)$ & .134 & \\
\hline Abiturnote & 2.36 & 0.55 & 2.51 & 0.53 & 5.28 & $(1,857)$ & $<.001$ & .28 \\
\hline Studienwahlmotivation intrinsisch & 4.84 & 0.56 & 4.77 & 0.61 & -2.25 & $(763)$ & .025 & .12 \\
\hline Studienwahlmotivation extrinsisch & 3.03 & 0.70 & 3.09 & 0.76 & 1.34 & $(1,670)$ & .179 & \\
\hline
\end{tabular}

Anmerkungen: GS Grundschule, HRS Haupt-und Realschule.

2008 bis Wintersemester 2011/2012) zu verschiedenen Messzeitpunkten zu ihren Studienvoraussetzungen und zu ihrem Studienverlauf befragt. Die Studienwahlmotivation wurde zu Studienbeginn erhoben. Die Befragungen zu Beginn des Studiums wurden im Rahmen von einführenden Vorlesungen papierbasiert durchgeführt, um hohe Rücklaufquoten zu erzielen. Zur Beantwortung der Forschungsfragen werden darüber hinaus a) die Noten zum Ende des Studiums herangezogen, welches durchschnittlich nach 9.86 Semestern erreicht wurde $(S D=3.32)$ und b) die kumulierten Noten über den Studienverlauf. Die zum Studienabschluss erfassten Noten wurden im Rahmen einer Befragung von den Studierenden selbst berichtet. $\mathrm{Zu}$ dieser online administrierten Befragung wurden jedes Semester diejenigen Studierenden eingeladen, die sich zur Ersten Staatsprüfung angemeldet hatten. Die kumulierten Noten setzen sich aus den im Studienverlauf erzielten Noten zusammen. Diese wurden der Forschergruppe vom Prüfungsbüro in einem pseudonymisierten Verfahren übermittelt.

\section{Stichprobe}

Aus der Gesamtstichprobe mit 1,969 Studierenden werden nur die Daten derer genutzt, von denen die selbstberichte Abschlussnote $(N=359)$ oder die kumulierten Noten im Studienverlauf $(N=403)$ vorliegen. 464 Studierenden kann mindestens eine dieser Noten zugeordnet werden. Von 298 Studierenden liegen beide Angaben vor. $\mathrm{Zu}$ Studienbeginn waren jene Studierenden, von denen eine Note vorliegt, durchschnittlich 21.1 Jahre alt $(S D=3.84)$. Ein Großteil dieser Studierenden ist weiblich (82.9\%). Mit rund einem Viertel studieren die wenigsten Haupt- und Realschullehramt (24.1\%), während die Gruppen der Grundschul- (38.0\%) und Gymnasiallehramtsstudierenden (38.0\%) etwas stärker vertreten sind.
Da im Vergleich zur Gesamtstichprobe nur rund einem Viertel der zu t1 befragten Studierenden eine Note zum Studienende zugeordnet werden konnte (23.6\%), wird zunächst ein Vergleich zwischen dieser Substichprobe und den übrigen Studierenden der Gesamtstichprobe vorgenommen (Dropout-Analyse; Tabelle 1). Ein Vergleich dieser beiden Studierendengruppen offenbart einige Unterschiede. In der hier untersuchten Substichprobe sind die weiblichen Studierenden signifikant häufiger vertreten als in der Gruppe jener Studierenden, von denen keine Noten vorliegen $\left(\chi^{2}(1)=37.14, p<.001\right)$. Auch mit Blick auf den Studiengang bestehen signifikante Unterschiede $\left(\chi^{2}(2)=31.19\right.$, $p<.001)$ : Während der Anteil Studierender für das Lehramt an Haupt- und Realschulen zwischen den Gruppen vergleichbar ist, sind die Grundschullehramtsstudierenden in der Substichprobe mit Noten häufiger vertreten, wohingegen die Studierenden des Gymnasiallehramts unterrepräsentiert sind. Zugleich weisen die Studierenden der hier untersuchten Substichprobe im Vergleich zu den übrigen Studierenden bessere Noten in ihrer Hochschulzugangsberechtigung auf $(t(1,857)=5.28, p<.001, d=0.28)$. Ein Vergleich der Studienwahlmotivation zeigt, dass sich die Studierendengruppen mit vs. ohne Noten zwar nicht in der Ausprägung extrinsischer Studienwahlmotivation unterscheiden, jedoch signifikante, wenngleich praktisch kaum bedeutsame, Unterschiede in der intrinsischen Studienwahlmotivation bestehen $(t(763)=-2.25, p=.03$, $d=0.12$ ).

\section{Messung der Studienwahlmotivation}

Zur Erfassung der Studienwahlmotivation wurden zu Beginn des Studiums die 33 Items des Fragebogens zur Erfassung der Motivation für die Wahl des Lehramtsstudiums (FEMOLA; Pohlmann \& Möller, 2010) verwendet. Die Items wurden mit folgendem Prompt versehen: „Ich habe 
Tabelle 2. Skalen zur Erfassung der Studienwahlmotivation

\begin{tabular}{|c|c|c|c|c|}
\hline Skala (Itemanzahl) & $\begin{array}{l}\text { Beispielitem } \\
\text { Ich habe das Lehramtsstudium gewählt, weil ... }\end{array}$ & $\alpha$ & M & $S D$ \\
\hline Intrinsische Studienwahlmotivation & & .83 & 4.85 & 0.55 \\
\hline Fachliches Interesse (5) & ich viel in meinen Fächern dazu lernen möchte. & .84 & 4.71 & 0.90 \\
\hline Pädagogisches Interesse (6) & $\begin{array}{l}\text { ich einen Beruf ausüben möchte, in dem ich die Erziehung } \\
\text { von Kindern und Jugendlichen mitgestalten kann. }\end{array}$ & .76 & 5.20 & 0.60 \\
\hline Fähigkeitsüberzeugung (5) & $\begin{array}{l}\text { mir schon häufiger rückgemeldet wurde, Dinge verständlich } \\
\text { erklären zu können. }\end{array}$ & .80 & 4.63 & 0.76 \\
\hline Extrinsische Studienwahlmotivation & & .88 & 3.37 & 0.77 \\
\hline Geringe Schwierigkeit (4) & das Studium nicht so anstrengend ist. & .79 & 2.01 & 0.79 \\
\hline Familiäre Nützlichkeit (4) & $\begin{array}{l}\text { ich auch neben dem Beruf noch Zeit für Familie, Freunde } \\
\text { und Hobbies haben will. }\end{array}$ & .83 & 4.17 & 1.11 \\
\hline Finanzielle Nützlichkeit (3) & ich als Lehrer/in finanziell abgesichert bin. & .87 & 4.08 & 1.19 \\
\hline Soziale Einflüsse (5) & $\begin{array}{l}\text { ich denke, dass meine Familie und meine Freunde } \\
\text { den Lehrerberuf als am besten geeignet für mich halten. }\end{array}$ & .76 & 3.18 & 1.08 \\
\hline
\end{tabular}

das Lehramtsstudium gewählt, weil ...“. Die Studierenden machten Angaben auf einem sechsstufigen Antwortformat (1 trifft gar nicht $z u$ bis 6 trifft völlig $z u) .{ }^{1}$ Mit dem FEMOLA wird die intrinsische Studienwahlmotivation über drei Skalen erfragt - über das Fachliche Interesse, das Pädagogische Interesse sowie die Fähigkeitsüberzeugung. Die extrinsische Studienwahlmotivation wird über die angenommene Geringe Schwierigkeit des Studiums, über Soziale Einflüsse sowie Nützlichkeitsaspekte abgebildet. Die letztgenannte Skala lässt sich in Anlehnung an Retelsdorf und Möller (2012) über die beiden Subskalen familien-/freizeitbezogene und finanzielle Nützlichkeitsaspekte erfassen.

Diese siebenfaktorielle Struktur mit zwei Faktoren zweiter Ordnung (intrinsisch und extrinsisch) wurde mittels konfirmatorischer Faktoranalyse für die hier zugrundeliegende Stichprobe mit dem Programm Mplus 7.2 (Muthén \& Muthén, 2015) geprüft. Dabei wurden fehlende Werte über den Full-Information-Maximum-Likelihood-Algorithmus (FIML) geschätzt. Zur Einschätzung der Modellgüte werden der Comparative Fit Index (CFI), der Tucker Lewis Index (TLI), der Root Square of Error Approximation (RMSEA) sowie der Standardized Root Mean Square Residual (SRMR) herangezogen. Für den CFI sollte der Wert möglichst nahe 1 liegen, weshalb in der Literatur ein Cut-off-Kriterium bei 95 vorgeschlagen wird (Schermelleh-Engel, Moosbrugger \& Müller, 2003). Mitunter wird auch ein Wert von 90 noch als akzeptabel angesehen (Bentler \& Bonett, 1980). Dieses Kriterium wird auch für den TLI verwendet (Geiser, 2011). Der RMSEA-
Wert sollte möglichst gering sein, die Grenze für einen akzeptablen Fit liegt bei .08. Für den SRMR sollte der Wert ebenfalls gering ausfallen. Werte unter .05 belegen einen guten Fit, bei Werten zwischen .05 und .10 wird von einem akzeptablen Fit ausgegangen. Zudem sollte das Verhältnis von $\chi^{2}$-Wert zu Freiheitsgraden unter 3 liegen, um von einem akzeptablen Fit auszugehen (Schermelleh-Engel et al., 2003).

Das siebenfaktorielle Modell mit zwei Faktoren zweiter Ordnung konnte mit noch ausreichender Modellanpassung bestätigt werden $\left(\chi^{2}=861.94 ; \mathrm{df}=450 ; \chi^{2} / \mathrm{df}=1.92\right.$, $p<.001, \mathrm{CFI}=.92, \mathrm{TLI}=.92$, RMSEA $=.04$, SRMR $=.07$ ). Aufgrund mangelnder Güte wurde folgendes Item der Nützlichkeitsskala aus dem Itempool ausgeschlossen: „Ich habe das Lehramtsstudium gewählt, weil ich in keinem anderen Beruf so viel Ferien hätte wie im Lehrerberuf". Basierend auf inhaltlichen Überlegungen und den Modifikationsindizes wurden sechs Residualkorrelationen zugelassen.

Die Skalen zeigen je eine zufriedenstellende Güte $\left(\alpha \geq .76, r_{\text {it }} \geq .35\right)$. Tabelle 2 zeigt die deskriptiven Kennwerte der Faktoren erster und zweiter Ordnung. Für die intrinsische und die extrinsische Studienwahlmotivation wurden zum Zwecke der gleichen Gewichtung die Werte der Primärfaktoren aggregiert.

\section{Noten als Kriterium für Studienleistungen}

Als Maß für Studienleistungen werden die Noten der Studierenden herangezogen. Dabei handelt es sich einerseits

\footnotetext{
Da in einer Kohorte mit dem Projekt PaLea (Panel zum Lehramtsstudium; Bauer et al., 2010) kooperiert wurde und hierbei der FEMOLA vierstufig erhoben wurde ( 1 = trifft gar nicht zu bis 4 = trifft völlig zu), wurden alle Werte dieser Kohorte im Nachgang transformiert und an das sechsstufige Antwortformat angepasst.
} 
um die von den Studierenden selbstberichtete Abschlussnote sowie andererseits um die kumulierten Noten während des Studienverlaufs mit Ausnahme der Noten aus den Abschlussprüfungen.

Selbstberichtete Abschlussnote. Für selbstberichtete Noten kann generell von einer ausreichend validen Angabe ausgegangen werden. Verschiedene Studien fanden eine hohe Korrelation zwischen selbstberichteten und tatsächlich erreichten Noten (Frucot \& Cook, 1994; Sparfeldt, Buch, Rost \& Lehmann, 2008), insbesondere dann, wenn Durchschnittsnoten statt Einzelnoten abgefragt werden (Cole \& Gonyea, 2010; Kuncel, Credé \& Thomas, 2005). In der Hochschulforschung werden selbstberichtete Noten demnach häufig als zuverlässige Indikatoren für Studienleistungen oder den Studienerfolg herangezogen (z.B. Schiefele, Streblow, Ermgassen \& Moschner, 2003; Trapmann, 2008). Von den 464 Studierenden in der Stichprobe berichteten 359 Befragte ihre Note im Rahmen der Online-Befragung. Sie wurden gebeten, die Durchschnittsnote ihres Staatsexamens mit einer Nachkommastelle anzugeben. In Abhängigkeit vom studierten Lehramt ergeben sich signifikante Unterschiede in der selbstberichteten Note $(F(2,353)=6.77, p=.001$, $\left.\eta^{2}=.04\right) .^{2}$ Die beste, also die geringste Durchschnittsnote weisen die Gymnasiallehramtsstudierenden auf $(M=1.68, S D=0.44)$, gefolgt von den Grundschullehramtsstudierenden $(M=1.69, S D=0.36)$ und den Studierenden mit Haupt- und Realschullehramt $(M=1.86$, $S D=0.35$ ). Die Scheffé Post-hoc-Tests belegen, dass sich die Studierenden des Haupt- und Realschullehramts signifikant von den Grund- ( $p=.008)$ sowie den Gymnasiallehramtsstudierenden $(p=.003)$ unterscheiden. Daneben bestehen auch geschlechtsspezifische Unterschiede $(t(356)=-3.58, p<.001, d=0.49)$ : Die weiblichen Studierenden $(M=1.69, S D=0.38)$ berichten im Vergleich zu den männlichen Studierenden $(M=1.89, S D=0.44)$ bessere Durchschnittsnoten.

Kumulierte Noten. Dieser Indikator für Studienleistungen setzt sich aus den im Studienverlauf erworbenen Noten zusammen. Die Gewichtung der Noten erfolgt entsprechend der jeweiligen Modulprüfungsordnungen der Studierenden. In die Berechnungen gehen folglich nicht die Noten aller Module ein, sondern jene, die auch in die Berechnung der Gesamtnote nach Abschluss des Studiums einfließen. Auf diese Weise errechnen sich die No- ten für den bildungswissenschaftlichen Anteil des Lehramtsstudiums $^{3}$ und die Fachnoten, welche gewichtet die kumulierten Noten im Studienverlauf ergeben. In diesem kumulierten Wert nicht enthalten sind die Noten der Abschlussprüfungen (hierzu zählen die Wissenschaftliche Hausarbeit, Klausuren und mündliche Abschlussprüfungen), welche insgesamt $40 \%$ der Gesamtnote ausmachen. Die kumulierten Noten liegen in Notenpunkten vor (theoretischer Range: 5-15 Punkte) ${ }^{4}$, wurden jedoch zur besseren Vergleichbarkeit in Noten transformiert, sodass für die Interpretierbarkeit der beiden Notenindikatoren gilt: Je niedriger der Wert, desto besser die Noten. Insgesamt liegen von 403 der 468 Studierenden der Stichprobe kumulierte Noten im Studienverlauf vor $(M=2.11, S D=0.45)$. Diese fallen im Vergleich zur selbstberichteten Note $(M=1.73, S D=0.40)$ schlechter aus. Auch für dieses Maß der Studienleistungen bestehen signifikante Unterschiede zwischen den Lehrämtern $\left(F_{\mathrm{BF}}(2,351.60)=10.78\right.$, $\left.p<.001, \eta^{2}=.05\right) .{ }^{5}$ Post-hoc-Tests (Games-Howell) zeigen, dass die Grundschullehramtsstudierenden $(M=2.00$, $S D=0.37)$ sowohl im Vergleich zu den Studierenden des Haupt- und Realschullehramts $(M=2.24, S D=0.41)$ signifikant besser abschneiden $(p<.001)$ als auch gegenüber den Studierenden des Gymnasiallehramts $(M=2.16$, $S D=0.52)$ signifikant bessere Noten aufweisen $(p=.004)$. Zudem bestehen erneut geschlechtsspezifische Unterschiede $(t(400)=-4.45, p<.001, d=0.58)$ zugunsten der weiblichen Studierenden (w: $M=2.07, S D=0.44, \mathrm{~m}$ : $M=2.33, S D=0.45)$.

\section{Umgang mit fehlenden Werten}

Wie für Panelstudien üblich, fehlen in dem zugrundeliegenden Subsample einige Werte. Der Anteil fehlender Werte schwankt für die zu analysierenden Variablen zwischen $10 \%$ und $23 \%$. Um die Teilstichprobengröße zu erhalten, wurden fehlende Werte mit dem Programm R 3.6.1 und dem Paket mice (Buuren \& Groothuis-Oudshoorn, 2011) multipel imputiert. Insgesamt wurden 20 Datensätze generiert. Zur Schätzung der Modelle wurden neben den Faktoren des FEMOLA sowie den Kontrollvariablen auch persönlichkeitsbezogene und motivationale Merkmale (z.B. Big Five: Hartig, Jude \& Rauch, 2003; LMI: Schuler, Prochaska \& Frintup, 2001) sowie Lernstrategien (LIST: Schiefele \& Wild, 1994) zu allen verfügbaren Messzeitpunkten herangezogen. Die folgen-

\footnotetext{
Weder die selbstberichtete Abschlussnote noch die kumulierten Noten sind normalverteilt. Aufgrund der Robustheit von t-Test und Varianzanalyse (Eid, Gollwitzer \& Schmitt, 2017) werden diese Verfahren trotz der Verletzung dieser Voraussetzung durchgeführt.

In diese Note fließt auch eine Note aus dem praktischen Teil der Ausbildung ein. Während die Unterrichtsversuche unbenotet sind, wird der Praktikumsbericht benotet.

${ }^{4}$ Unterhalb von 5 Notenpunkten gilt eine Prüfung als nicht bestanden. Der angegebene Range bezieht sich auf Leistungen bestandener Modulprüfungen, die Voraussetzungen für das Absolvieren des Staatsexamens sind.

Aufgrund der Verletzung der Homogenitätsannahme werden in diesem Abschnitt Korrekturen nach Brown-Forsythe und Games-Howell gewählt (Eid et al., 2017).
} 
Tabelle 3. Bivariate Zusammenhänge zwischen Primär- und Sekundärfaktoren der Studienwahlmotivation und Noten

\begin{tabular}{|c|c|c|c|c|c|c|c|c|c|c|c|c|}
\hline & & 1 & 2 & 3 & 4 & 5 & 6 & 7 & 8 & 9 & 10 & 11 \\
\hline 1 & Intrinsische Studienwahlmotivation & & & & & & & & & & & \\
\hline 2 & Extrinsische Studienwahlmotivation & .15 & & & & & & & & & & \\
\hline 3 & Fachliches Interesse & .77 & .15 & & & & & & & & & \\
\hline 4 & Pädagogisches Interesse & .62 & .00 & .17 & & & & & & & & \\
\hline 5 & Fähigkeitsüberzeugung & .78 & .14 & .35 & .35 & & & & & & & \\
\hline 6 & Geringe Schwierigkeit & -.03 & .62 & .03 & -.09 & -.03 & & & & & & \\
\hline 7 & Soziale Einflüsse & .16 & .67 & .09 & .10 & .16 & .30 & & & & & \\
\hline 8 & $\begin{array}{l}\text { Nützlichkeit: Vereinbarkeit von Familie } \\
\text { und Beruf }\end{array}$ & .11 & .75 & .09 & .04 & .10 & .33 & .24 & & & & \\
\hline 9 & Nützlichkeit finanzielle Sicherheit & .14 & .79 & .18 & -.06 & .14 & .30 & .32 & .51 & & & \\
\hline 10 & Selbstberichtete Abschlussnote & -.20 & .11 & -.10 & -.14 & -.20 & .07 & .05 & .08 & .12 & & \\
\hline 11 & Kumulierte Noten im Studienverlauf & -.09 & .14 & .00 & -.06 & -.14 & .15 & .10 & .04 & .13 & .69 & \\
\hline 12 & Note der Hochschulzugangsberechtigung & -.04 & .07 & -.03 & .10 & -.12 & .02 & .06 & .03 & .07 & .36 & .38 \\
\hline
\end{tabular}

Anmerkung: Signifikante Koeffizienten sind fett gedruckt $(p<.05)$.

den Analysen wurden mit den imputierten Datensätzen durchgeführt.

\section{Analyseverfahren}

Die prädiktive Kraft der Studienwahlmotivation für die Studienleistungen - operationalisiert über die selbstberichtete Abschlussnote sowie die kumulierten Noten im Studienverlauf - wird unter Nutzung von Mplus 7.2 (Muthén \& Muthén, 2015) untersucht, da im Programm auch für die gepoolten Ergebnisse die standardisierten Regressionskoeffizienten sowie $R^{2}$ ausgegeben werden. Zudem kann ein gegen Verletzungen der Normalverteilungsannahmen robuster Maximum-Likelihood Schätzer ausgewählt werden (mlr Schätzer). Zur Beantwortung der Forschungsfragen werden multiple lineare Regressionen mit manifesten Variablen berechnet. Dabei wird zweischrittig vorgegangen: Zunächst werden zur Beantwortung der Forschungsfragen I bis $I V$ die Effekte der Sekundärfaktoren auf die beiden Merkmale der Studienleistungen überprüft. Daran schließt sich eine simultane Schätzung der Effekte der Primärfaktoren auf die beiden Merkmale der Studienleistungen an (Forschungsfragen $V$ und VI). Dabei werden je zwei Modelle spezifiziert, die sich ausschließlich in der abhängigen Variablen - dem Maß für den Studienerfolg - unterscheiden. Die Analysen erfolgen unter Kontrolle von Geschlecht und Lehramt, da die Noten in Abhängigkeit dieser Merkmale variieren. Darüber hinaus wird die Abiturnote als Kontrollvariable aufgenommen, da diese als bedeutsamer Prädiktor für den Studienerfolg gilt (z. B. Wolf, Kunina-Habenicht, Maurer \& Kunter, 2018).

\section{Ergebnisse}

\section{Bivariate Zusammenhänge}

Tabelle 3 gibt einen Überblick über die bivariaten Zusammenhänge der intervallskalierten Variablen. Zwischen der intrinsischen und extrinsischen Studienwahlmotivation besteht ein geringer positiver Zusammenhang $(r=.15$, $p=.003)$. Die selbstberichtete Abschlussnote und die kumulierten Noten im Studienverlauf hängen hoch miteinander zusammen $(r=.69, p<.001)$. Darüber hinaus geben die Korrelationen bereits einen Hinweis auf die interessierenden Zusammenhänge zwischen der Studienwahlmotivation und den im Studium erzielten Noten. Die intrinsische Studienwahlmotivation zeigt negative Zusammenhänge mit der selbstberichteten Abschlussnote $(r=-.20, p<.001)$ sowie den kumulierten Noten im Studienverlauf $(r=-.09$, $p=$.073). Mit einer stärkeren intrinsischen Studienwahlmotivation zu Studienbeginn gehen demnach niedrigere, also bessere Noten zum Studienende einher. Jedoch fällt nur der Zusammenhang mit der selbstberichteten $\mathrm{Ab}$ schlussnote signifikant aus. Für die extrinsische Studienwahlmotivation bestehen hingegen positive Zusammenhänge mit der selbstberichteten Abschlussnote $(r=.11$, $p=.018)$ sowie den kumulierten Noten im Studienverlauf $(r=.15, p=.003)$. Die positiven Ausprägungen der Zusammenhänge weisen darauf hin, dass mit einer höheren extrinsischen Studienwahlmotivation schlechtere Noten verbunden sind. Für die verschiedenen Primärfaktoren zeigen sich sowohl mit Blick auf die selbstberichtete Abschlussnote als auch mit Blick auf die kumulierten Noten im Studienverlauf differentielle Zusammenhänge. 


\section{Vorhersagekraft der Studienwahlmotivation für die Studienleistungen}

Um die Fragestellung I nach dem Einfluss der intrinsischen Studienwahlmotivation sowie die Fragestellung II nach dem Einfluss der extrinsischen Studienwahlmotivation auf die selbstberichtete Abschlussnote zu beantworten, können die Ergebnisse von Modell 1 (Tabelle 4) herangezogen werden. Unter Kontrolle von Geschlecht, Lehramt und Abiturnote besteht ein signifikant negativer Einfluss der intrinsischen Studienwahlmotivation zu Beginn des Studiums auf die selbstberichtete Abschlussnote $(\beta=-.20$, $p=.004)$. Mit einer höheren intrinsischen Studienwahlmotivation geht folglich eine bessere selbstberichtete Abschlussnote einher. Wenngleich der Zusammenhang nur gering ausgeprägt ist, kann Hypothese 1 bestätigt werden. Auch die extrinsische Studienwahlmotivation beeinflusst unter Berücksichtigung der Kontrollvariablen schwach die selbstberichtete Abschlussnote $(\beta=.12, p=.013)$. Im Gegensatz zur intrinsischen Motivation zeigt sich jedoch ein positiver Einfluss, sodass mit einer höheren extrinsischen Motivation eine schlechtere selbstberichtete Abschlussnote assoziiert ist. Die intrinsische und extrinsische Studienwahlmotivation erklären über die Kontrollvariablen hinaus rund $4 \%$ der Varianz der selbstberichteten $\mathrm{Ab}$ schlussnote. Von den Kontrollvariablen erweisen sich sowohl die Abiturnote als auch das Geschlecht als signifikante Prädiktoren.

Modell 2 in Tabelle 4 liefert Antworten auf die Forschungsfragen III und IV, mit welchen die Vorhersagekraft der intrinsischen Studienwahlmotivation und der extrin-

Tabelle 4. Vorhersage der selbstberichten Abschlussnoten und der kumulierten Noten durch die Sekundärfaktoren der Studienwahlmotivation

\begin{tabular}{|c|c|c|c|c|c|c|}
\hline & \multicolumn{3}{|c|}{$\begin{array}{c}\text { Modell } 1 \\
\text { (AV: selbstberichtete } \\
\text { Abschlussnote) }\end{array}$} & \multicolumn{3}{|c|}{$\begin{array}{c}\text { Modell } 2 \\
\text { (AV: kumulierte Noten } \\
\text { gesamt) }\end{array}$} \\
\hline & $\beta$ & $S E$ & $p$ & $\beta$ & $S E$ & $p$ \\
\hline \multicolumn{7}{|l|}{ Kontrollvariablen } \\
\hline Geschlecht & .12 & .05 & .007 & .13 & .05 & .006 \\
\hline Lehramt GS & .01 & .05 & .863 & -.18 & .05 & $<.001$ \\
\hline Lehramt HRS & .05 & .05 & .380 & -.05 & .05 & .380 \\
\hline Abiturnote & .31 & .06 & $<.001$ & .35 & .05 & $<.001$ \\
\hline \multicolumn{7}{|c|}{ Studienwahlmotivation } \\
\hline Intrinsisch & -.20 & .07 & .004 & -.10 & .05 & .020 \\
\hline \multirow[t]{2}{*}{ Extrinsisch } & .12 & .05 & .013 & .10 & .05 & .032 \\
\hline & \multicolumn{3}{|c|}{$R^{2}=.19, p<.001 ; \Delta R^{2}=.04$} & \multicolumn{3}{|c|}{$R^{2}=.22, p<.001 ; \Delta R^{2}=.02$} \\
\hline
\end{tabular}

Anmerkungen: Geschlecht: 1 weiblich, 2 männlich; Lehramt GS: 0 kein Lehramt Grundschule, 1 Lehramt Grundschule, Lehramt HRS: 0 kein Lehramt Haupt- und Realschule, 1 Lehramt Haupt- und Realschule; $\triangle R^{2}$ : Veränderung $R^{2}$ durch Aufnahme der Sekundärfaktoren der Studienwahlmotivation. sischen Studienwahlmotivation für die kumulierten Noten im Studienverlauf geprüft wird. Wie schon im Modell 1, besteht auch im Modell 2 ein schwacher, signifikanter Einfluss der intrinsischen $(\beta=-.10, p=.020)$ wie auch der extrinsischen $(\beta=.10, p=.032)$ Studienwahlmotivation auf die Studienleistungen. Damit kann Hypothese 2 bestätigt werden: Je stärker die Studierenden intrinsisch motiviert waren für ihr Studium, desto besser sind ihre Leistungen im Verlauf des Studiums. Der positive Einfluss der extrinsischen Studienwahlmotivation auf die tatsächlich im Studienverlauf erhaltenen Noten verweist darauf, dass mit einer höheren extrinsischen Studienwahlmotivation schlechtere Noten im Studium einhergehen. Über die Kontrollvariablen hinaus kann die Studienwahlmotivation allerdings nur einen geringen Beitrag zur Varianzaufklärung der abhängigen Variable leisten $\left(\Delta R^{2}=.02\right)$.

Den insgesamt stärksten Prädiktor stellt jeweils die Abiturnote dar (Modell 1: $\beta=.31, p<.001 ;$ Modell 2: $\beta=.35$, $p<.001)$. Daneben nimmt auch das Geschlecht Einfluss auf die selbstberichtete Abschlussnote und die kumulierten Noten im Studium (Modell 1: $\beta=.12, p=.007$; Modell 2: $\beta=.13, p=.006$ ). Die Lehramtsstudentinnen weisen im Vergleich zu den -studenten bessere Noten zum Studienende auf. Das studierte Lehramt beeinflusst zudem die kumulierten Noten im Studium (Modell 2: Lehramt GS $\beta=-.18, p<.001$ ).

Die Modelle 3 und 4 liefern schließlich Informationen über die Vorhersagekraft der Primärfaktoren der Studienwahlmotivation (Fragestellungen $V$ und VI; Tabelle 5). Bemerkenswerte Ergebnisse ergeben sich bei einem vergleichenden Blick auf die beiden Modelle: Während keiner der Primärfaktoren auf die selbstberichtete Abschlussnote wirkt, und damit die Hypothesen 3 bis 5 verworfen werden müssen, können die kumulierten Noten im Studienverlauf durch die Fähigkeitsüberzeugung $(\beta=-.12, p=.039)$ als Faktor der intrinsischen Motivation erklärt werden, nicht jedoch durch das Pädagogische oder das Fachliche Interesse. Damit lässt sich Hypothese 7 bestätigen, während die Hypothesen 6 und 8 verworfen werden müssen. Interessant ist, dass die kumulierten Noten neben der Fähigkeitsüberzeugung auch durch die wahrgenommene geringe Schwierigkeit des Studiums erklärt werden können, welche einen signifikant positiven Effekt zeigt ( $\beta=.10, p=.038)$.

Durch Hinzunahme der Primärfaktoren der Studienwahlmotivation kann deskriptiv etwas mehr Varianz erklärt werden als durch Hinzunahme der Sekundärfaktoren (Modell 3: $\Delta R^{2}=.05 ;$ Modell 4: $\Delta R^{2}=.04$ ). 
Tabelle 5. Vorhersage der selbstberichten Abschlussnoten und der kumulierten Noten durch die Primärfaktoren der Studienwahlmotivation

\begin{tabular}{|c|c|c|c|c|c|c|}
\hline & \multicolumn{3}{|c|}{$\begin{array}{c}\text { Modell } 3 \\
\text { (AV: selbstberichtete } \\
\text { Abschlussnote) }\end{array}$} & \multicolumn{3}{|c|}{$\begin{array}{c}\text { Modell } 4 \\
\text { (AV: kumulierte Noten } \\
\text { gesamt) }\end{array}$} \\
\hline & $\beta$ & $S E$ & $p$ & $\beta$ & $S E$ & $p$ \\
\hline \multicolumn{7}{|l|}{ Kontrollvariablen } \\
\hline Geschlecht & .12 & .05 & .008 & .13 & .05 & .006 \\
\hline Lehramt GS & .05 & .06 & .402 & -.19 & .06 & .001 \\
\hline Lehramt HRS & .07 & .05 & .226 & -.06 & .05 & .244 \\
\hline Abiturnote & .32 & .06 & $<.001$ & .35 & .05 & $<.001$ \\
\hline \multicolumn{7}{|l|}{ Studienwahlmotivation } \\
\hline Pädagogisches Interesse & -.13 & .07 & .072 & .00 & .05 & .989 \\
\hline Fähigkeitsüberzeugung & -.11 & .06 & .082 & -.12 & .06 & .039 \\
\hline Fachliches Interesse & -.04 & .05 & .491 & .00 & .05 & .938 \\
\hline Geringe Schwierigkeit & -.01 & .05 & .909 & .10 & .05 & .038 \\
\hline Soziale Einflüsse & .03 & .06 & .558 & .06 & .05 & .277 \\
\hline Familiäre Nützlichkeit & .06 & .06 & .257 & .00 & .05 & .960 \\
\hline \multirow{2}{*}{ Finanzielle Nützlichkeit } & .06 & .06 & .216 & .01 & .06 & .885 \\
\hline & \multicolumn{3}{|c|}{$R^{2}=.20, p<.001 ; \Delta R^{2}=.05$} & \multicolumn{3}{|c|}{$R^{2}=.23, p<.001 ; \Delta R^{2}=.04$} \\
\hline
\end{tabular}

Anmerkungen: Geschlecht: 1 weiblich, 2 männlich; Lehramt GS: 0 kein Lehramt Grundschule, 1 Lehramt Grundschule, Lehramt HRS: 0 kein Lehramt Haupt- und Realschule, 1 Lehramt Haupt- und Realschule; $\triangle R^{2}$ : Veränderung $R^{2}$ durch Aufnahme der Faktoren der Studienwahlmotivation.

\section{Diskussion}

Zwar wurde die Studienwahlmotivation Lehramtsstudierender vielfach untersucht, die prädiktive Kraft auf die Leistungen im Studium wurde mithilfe längsschnittlicher Studiendesigns bislang jedoch kaum geprüft. Vor diesem Hintergrund wurde in der vorliegenden Studie die Vorhersagekraft der Studienwahlmotivation zu Beginn des Studiums für Studienleistungen - operationalisiert über die selbstberichtete Abschlussnote und über die kumulierten Noten - in den Blick genommen. Über die bisherigen Untersuchungen hinausgehend, wurde der Einfluss der Studienwahlmotivation im Längsschnitt - über das gesamte Lehramtsstudium - und über verschiedene Lehramtsstudiengänge hinweg geprüft. Zudem wurden neben den selbstberichteten Noten auch extern erfasste Noten im Studium als Kriterium herangezogen. Im Kern ergeben sich schwache Zusammenhänge, die theoretisch erklärbar sind: Je stärker Studierende zu Beginn intrinsisch für ihr Studium motiviert waren, desto besser sind ihre Noten, je stärker sie extrinsisch motiviert waren, desto schlechter fallen die Noten aus. Im Folgenden werden die Ergebnisse im Detail diskutiert.

Aufgrund theoretischer Annahmen (z.B. Kunter, 2014; Rothland, 2014) und empirischer Befunde (z.B. Brühwiler, 2001; Cramer, 2012; Mayr, 2009) wurden signifi- kant positive Einflüsse der Faktoren intrinsischer Studienwahlmotivation auf die selbstberichtete Abschlussnote sowie die kumulierten Noten im Studienverlauf angenommen. Diese Annahme lässt sich für die selbstberichtete Abschlussnote jedoch lediglich auf der aggregierten Ebene des Sekundärfaktors bestätigen. Ein etwas anderes Bild ergibt sich, wenn das Kriterium der kumulierten Noten betrachtet wird: Je ausschlaggebender die Fähigkeitsüberzeugung für die Studienwahlmotivation zu Beginn des Lehramtsstudiums war, desto besser fallen die kumulierten Noten aus, ein Befund, der sich auch mit der allgemeinen Selbstkonzeptforschung erklären lässt: Leistungen profitieren vom Zutrauen in die eigenen Fähigkeiten (z.B. Möller \& Trautwein, 2015). Demgegenüber erweisen sich weder das pädagogische noch das fachliche Interesse als vorhersagerelevant. Die kumulierten Noten hängen zudem auch mit der antizipierten Schwierigkeit des Studiums zusammen. Studierende, die das Lehramtsstudium gewählt haben, weil sie es als einfach einschätzten, erbringen im Studium schlechtere Leistungen als jene, die dies weniger stark als Grund für die Studienwahl angaben. Dieser Effekt tritt nicht auf, wenn man die selbstberichtete Abschlussnote als Kriterium heranzieht. Wie lässt sich dieser Unterschied in der prädiktiven Kraft dieses extrinsischen Faktors erklären? Möglicherweise korrigieren jene Studierende, die im Verlauf des Studiums merken, dass sie die Anforderungen unterschätzt haben, ihr Lernverhalten, sodass sie zum Abschluss bessere Noten erreichen und der Zusammenhang zwischen der antizipierten Schwierigkeit des Studiums zu Studienbeginn und der Leistung am Studienende dann nicht mehr signifikant wird. Denn die Abschlussphase stellt einen besonderen Studienabschnitt dar, der eine mögliche Unterschätzung sichtbar machen kann und Sozialisations- und Anpassungseffekte auslösen könnte: Eine relative Freiheit bei der Themenwahl der Abschlussarbeit und weiterer Prüfungen könnte derartige Entwicklungen durchaus begünstigen. Überraschend ist in diesem Zusammenhang allerdings, dass sich keine der Noten durch fachliches und pädagogisches Interesse vorhersagen lässt. Dieses erwartungswidrige Ergebnis lässt sich eventuell damit erklären, dass die eingesetzten Items nicht differenziert das fachliche Interesse für jedes studierte Fach erfragen, aber davon ausgegangen werden muss, dass sich das Interesse an den Fächern unterscheidet. $\mathrm{Zu}$ berücksichtigen ist dabei einerseits, dass Grundschullehramtsstudierende kaum 
Wahlmöglichkeiten haben, sondern in Hessen die Fächer Mathematik und Deutsch belegen müssen. Andererseits sind einige Fächer zulassungsbeschränkt, sodass eine Fächerwahl nicht immer interessengeleitet erfolgen kann. Theoretisch erwartbare Zusammenhänge könnten durch diese Mechanismen abgeschwächt werden. Ähnlich kann der ausbleibende Effekt des pädagogischen Interesses interpretiert werden. Die beispielsweise in den Items beschriebene Freude an der Arbeit mit Kindern und Jugendlichen könnte zu weit von den meisten Studieninhalten entfernt sein, als dass dieser Faktor alleine bedeutsam Einfluss auf (aggregierte) Leistungen bis zum Ende des Studiums nimmt. In Kombination mit hohem fachlichem Interesse und einer hohen Fähigkeitsüberzeugung werden diese längerfristigen Wirkungen wiederum sichtbar: Betrachtet man die Ebene der Sekundärfaktoren, erwies sich die intrinsische Studienwahlmotivation - erwartungskonform - als bedeutsamer Prädiktor für die herangezogenen Leistungsmaße. Es zeigt sich, dass mit einer höheren intrinsischen Studienwahlmotivation zu Studienbeginn bessere Noten zum Studienende einhergehen. Dabei war der Einfluss auf die selbstberichte Abschlussnote $(\beta=-.20, p=.004)$ noch etwas höher als der Einfluss auf die kumulierten Noten im Studienverlauf $(\beta=-.10$, $p=.020)$. Analog zu den Ergebnissen von Mayr (2009) sind mit einer höheren extrinsischen Motivation zu Studienbeginn schlechtere Noten zum Studienende verbunden, hier jedoch in den Noten des Ersten Staatsexamens insgesamt und nicht - wie bei Mayr (2009) - in der Note für die schriftliche Abschlussarbeit. Zudem gehen mit einer höheren extrinsischen Motivation am Anfang des Studiums schwächere Noten im Studienverlauf einher. Die Koeffizienten zeigen für die selbstberichtete Note $(\beta=.12$, $p=.013)$ und die kumulierten Noten im Studienverlauf vergleichbare Ausprägungen $(\beta=.10, p=.032)$. Durch die Aggregierung der Daten auf der Sekundärebene scheinen Wirkungen intrinsischer und extrinsischer Motivationsfacetten stärker zutage zu treten. Dabei muss allerdings bedacht werden, dass über den mehrjährigen Untersuchungszeitraum insgesamt nur wenig Varianz in den Kriterien durch die unabhängigen Variablen erklärbar ist. Der stärkste Prädiktor ist in allen Modellen die Abiturnote, was erwartet werden konnte (z.B. Trapmann, 2008; Wolf et al., 2018). Neben den Kontrollvariablen erklärt die Studienwahlmotivation nur 2 bis $5 \%$ der Varianz der Noten im Studienverlauf. Allerdings muss angemerkt werden, dass ausgehend von der existierenden Literatur sowie angesichts des großen zeitlichen Abstands kaum deutlichere Zusammenhänge erwartbar sind (z. B. König \& Rothland, 2012, 2013). Die Erklärungskraft könnte möglicherweise erhöht werden, indem die Kriterien getrennt nach den Studienbereichen des Lehramts operationalisiert werden: So ist durchaus vorstellbar, dass die Fak- toren der Studienwahlmotivation unterschiedlich auf fachwissenschaftliche, fachdidaktische und bildungswissenschaftliche und schulpraktische Leistungen wirken (s.a. Schwippert et al., 2013).

Die hier gefundenen Zusammenhänge zwischen der Studienwahlmotivation und den Noten im Studium können dennoch als ein Hinweis auf die prognostische Validität der mit den FEMOLA generierten Daten interpretiert werden. Die Studienwahlmotivation beeinflusst über die Dauer des Studiums zu substanziellen Anteilen den Studienerfolg. Dieser zentrale Befund hat auch Bedeutung für den zu erwartenden beruflichen Erfolg: Interpretiert man die hier herangezogenen Noten als Ausdruck kumulierter Wissensbestände, kann im Sinne des Modells professioneller Handlungskompetenz (Baumert \& Kunter, 2006) argumentiert werden, dass Lehrpersonen mit einer hohen intrinsischen und einer geringen extrinsischen Studienwahlmotivation auch größeren beruflichen Erfolg haben sollten. Umfassende Wissensbestände sollten es Lehrpersonen erleichtern, einen guten und damit lernwirksamen Unterricht zu gestalten (Baumert et al., 2010; Voss, Kunter, Seiz, Hoehne \& Baumert, 2014; s. a. Biermann et al., 2019).

In diese Richtung weisen auch Befunde von Richardson und Watt (2014). Sie untersuchten den Einfluss der Berufswahlmotivation auf das selbstberichtete unterrichtliche Verhalten im Beruf befindlicher Lehrpersonen. Neben dem wahrgenommenen gesellschaftlichen Nutzen sagt die Fähigkeitsüberzeugung, ein Faktor der intrinsischen Studien- bzw. Berufswahlmotivation, selbstberichtetes, positives Lehrerverhalten im Beruf vorher. Das Motiv der Verlegenheitslösung sowie die sozialen Einflüsse wirken sich hingegen ungünstig auf das Verhalten im Beruf aus. Zusammenfassend wird resümiert: „Our results highlight that initial motivations for choosing teaching do matter and impact dimensions of beginning teachers' anticipated professional engagement as they embark on their career, as well as self-reported teaching style up to eight years in teaching." (Richardson \& Watt, 2014, S.13). Darauf weisen auch weitere Forschungsbefunde hin: In der Untersuchung von Lipowsky (2003) erweist sich die retrospektiv, zum Zeitpunkt des Berufseinstiegs erfasste Studienwahlmotivation als bedeutsam für den erfolgreichen Übergang in den Lehrerberuf sowie für die berufliche $\mathrm{Zu}-$ friedenheit. Im weiteren Verlauf der Berufsbiografie der Lehrkräfte beeinflusst die zum Berufseinstieg erhobene Studienwahlmotivation auch den 11 Jahre später erfragten Enthusiasmus (Bleck, 2019). Mit diesen Ergebnissen belegen beide Untersuchungen eine hohe Bedeutsamkeit der intrinsischen Berufswahlmotivation, indem - auch unter Kontrolle weiterer Prädiktoren - langfristig positive Wirkungen intrinsischer Studien- bzw. Berufswahlmotivation empirisch nachgewiesen werden. 


\section{Limitationen und Ausblick}

Limitationen bestehen mit Blick auf die untersuchte Stichprobe. Da nur Studierende der Universität Kassel befragt wurden, ist die Übertragbarkeit der Befunde auf andere Universitäten und Pädagogische Hochschulen zu überprüfen, da standortspezifische Unterschiede in soziodemografischen Eingangsmerkmalen, Kriterien und den Motivlagen bestehen können. Zudem konnten nur die Daten einer Teilstichprobe der Erstsemesterstudierenden für die Analysen genutzt werden. Wie die Dropout-Analyse gezeigt hat, unterscheiden sich die selbstberichteten Abiturnoten der Studierenden in der Teilstichprobe von den Abiturnoten der Studierenden, die nicht in die Analysen einbezogen werden konnten (Tabelle 1). Es ist davon auszugehen, dass sich die Teilstichproben analog auch in ihren Studienleistungen unterscheiden (z.B. Wolf et al., 2018). Dies dürfte eine Varianzeinschränkung in den abhängigen Variablen mit sich bringen und könnte eine weitere Erklärung für die eher geringen Zusammenhänge sein.

Einschränkend muss außerdem berücksichtigt werden, dass mit der Studienwahlmotivation zu Studienbeginn ein eher indirekter Einflussfaktor untersucht wurde (König \& Rothland, 2012; Rothland, 2014). Wie die Lehramtsstudierenden die Lerngelegenheiten im Studium nutzten und welche Wirkmechanismen den Einfluss der Motivation auf den Studienerfolg erklären können, kann diese Studie nicht beantworten. Vor diesem Hintergrund ist aber bemerkenswert, dass motivationale Voraussetzungen der Studierenden zu Studienbeginn über eine durchschnittliche Dauer von fast 5 Jahren Einfluss auf den Studienerfolg nehmen. In bisherigen Untersuchungen fungierte u.a. die Lernzielorientierung als Mediator (König \& Rothland, 2013). Diese ist bei intrinsisch motivierten Studierenden ebenso wie die Leistungsmotivation höher ausgeprägt (Beckmann, 2016; Billich-Knapp et al., 2012; König \& Rothland, 2013). Auch Maße wie die Strategienutzung im Studium oder Persönlichkeitsmerkmale werden mitunter als vermittelnde Variablen zur Erklärung des Studienerfolgs herangezogen (Künsting \& Lipowsky, 2011; Trapmann, Hell, Hirn \& Schuler, 2007; s.a. Mertens, Sann, Böttger, Haase, Hannover \& Braun, 2020). Eine breitere Operationalisierung von Studienleistungen durch nicht-kognitive Maße wäre wünschenswert und zielführend, um sich dem Konstrukt Studienerfolg stärker anzunähern. In diesem Sinne erscheint es erstrebenswert, Indikatoren für Studienerfolg breiter zu konzeptualisieren. Neben objektiven Maßen (z.B. Tests zur Erfassung des bildungswissenschaftlichen oder fachspezifischen Wissens, Studiendauer, Noten für einzelne Module) sollten auch affektiv-motivationale Kriterien, wie die Studienzufriedenheit, die Selbstwirksamkeitserwartung oder die emotionale Erschöpfung der Lehramtsstudierenden berücksichtigt werden (Blömeke, 2009). Insgesamt kann mit solchen konzeptionellen Erweiterungen der abhängigen und unabhängigen Variablen trotz großer zeitlicher Abstände auch eine größere Varianzaufklärung erwartet werden.

Für zukünftige Untersuchungen wäre es zudem vorteilhaft, auf einer größeren Datenbasis Unterschiede zwischen den verschiedenen Lehramtsstudiengängen $\mathrm{zu}$ untersuchen. Über verschiedene Studien hinweg zeigt sich, dass in Abhängigkeit vom gewählten Lehramt Unterschiede in der Studienwahlmotivation bestehen. Dabei weisen in der Regel die Studierenden des Grundschullehramts ein höheres pädagogisches Interesse auf als Studierende des Gymnasiallehramts (Cramer, 2012; Retelsdorf \& Möller, 2012). Teils deuten sich auch deskriptive Unterschiede im fachlichen Interesse der Studierenden an, welches bei den Studierenden mit dem Ziel Gymnasiallehramt insgesamt höher ausgeprägt ist als bei den Studierenden anderer Lehrämter (Neugebauer, 2013; Retelsdorf \& Möller, 2012). Inwiefern sich nicht nur die Ausprägungen von Facetten der Motivation unterscheiden, sondern auch differentielle Zusammenhänge zwischen der Motivation und Studienerfolgsmaßen für die Lehrämter bestehen, ist bislang kaum untersucht. Hinweise auf entsprechende Interaktionen finden sich allerdings in der Studie von Künsting und Lipowsky (2011). Die Ergebnisse deuten darauf hin, dass der Zusammenhang zwischen intrinsischer und extrinsischer Motivation bei den Haupt- und Realschullehramtsstudierenden im Vergleich zu den Studierenden des Gymnasiallehramts signifikant stärker ist. Zudem wirkt sich die extrinsische Studienwahlmotivation bei den Studierenden des Haupt- und Realschullehramts signifikant positiv auf die Strategienutzung aus, nicht jedoch bei den anderen Lehrämtern. Inwieweit auch für den Einfluss der Studienwahlmotivation auf den Studienerfolg bzw. die Noten differentielle Zusammenhänge in Abhängigkeit vom studierten Lehramt bestehen, ist mit der zugrundeliegenden Teilstichprobe nicht statistisch abzusichern, soll aber in Folgestudien geprüft werden.

Trotz der aufgezeigten Einschränkungen befasst sich der Beitrag mit einem zentralen Forschungsdesiderat, da die Bedeutung der Studienwahlmotivation Lehramtsstudierender für Prozesse der Professionalisierung bislang nicht hinreichend untersucht wurde (Cramer, 2016b; Rothland, 2014). Dass sich in der vorliegenden Studie Zusammenhänge mit Leistungsmaßen bis zum Ende des Studiums in erwarteter Richtung ergeben, stellt einen Beleg für die Eignung des FEMOLA (Pohlmann \& Möller, 2010) dar, Beweggründe für die Wahl eines Lehramtsstudiums valide erfassen zu können.

\section{Literatur}

Bauer, J., Drechsel, B., Retelsdorf, J., Kauper, T., Rösler, L., Prenzel, M. \& Möller, J. (2010). Panel zum Lehramtsstudium - PaLea: Ent- 
wicklungsverläufe zukünftiger Lehrkräfte im Kontext der Reform der Lehrerbildung. Beiträge zur Hochschulforschung, 32, 34- 55.

Baumert, J. \& Kunter, M. (2006). Stichwort: Professionelle Kompetenz von Lehrkräften. Zeitschrift für Erziehungswissenschaft, 9 , 469 - 520. https://doi.org/10.1007/s11618-006-0165-2

Baumert, J., Kunter, M., Blum, W., Brunner, M., Voss, T., Jordan, A. et al. (2010). Teachers' mathematical knowledge, cognitive activation in the classroom, and student progress. American Educational Research Journal, 47, 133 - 180. https://doi.org/10.3102/ 0002831209345157

Beckmann, V. (2016). Studien- und Berufswahlmotive am Anfang des Lehramtsstudiums. In A. Boeger (Hrsg.), Eignung für den Lehrerberuf. Auswahl und Förderung (S.115-135). Wiesbaden: Springer VS.

Bentler, P. M. \& Bonett, D. G. (1980). Significance tests and goodness-of-fit in the analysis of covariance structures. Psychological Bulletin, 85, $588-600$.

Biermann, A., Dörrenbächer-Ulrich, L., Grassmé, I., Perels, F., Gläser-Zikuda, M. \& Brünken, R. (2019). Hoch motiviert, engagiert und kompetent: Eine profilanalytische Untersuchung zur Studien- und Berufswahlmotivation von Lehramtsstudierenden. Zeitschrift für Pädagogische Psychologie, 33, 177 - 189. https:// doi.org/10.1024/1010-0652/a000242

Billich-Knapp, M., Künsting, J. \& Lipowsky, F. (2012). Profile der Studienwahlmotivation bei Grundschullehramtsstudierenden. Zeitschrift für Pädagogik, 58, 696-719.

Bleck, V. (2019). Lehrerenthusiasmus. Entwicklung, Determinanten, Wirkungen. Wiesbaden: Springer.

Blömeke, S. (2009). Ausbildungs- und Berufserfolg im Lehramtsstudium im Vergleich zum Diplom-Studium - Zur prognostischen Validität kognitiver und psycho-motivationaler Auswahlkriterien. Zeitschrift für Erziehungswissenschaft, 12, 82-110. https://doi.org/10.1007/s11618-008-0044-0

Brookhart, S. M. \& Freeman, D. J. (1992). Characteristics of entering teacher candidates. Review of Educational Research, 62, 37-60. https://doi.org/10.3102/00346543062001037

Brühwiler, C. (2001). Die Bedeutung von Motivation in der Lehrerinnen- und Lehrerausbildung. In F. Oser \& J. Oelkers (Hrsg.), Die Wirksamkeit der Lehrerbildungssysteme. Von der Allrounderbildung zur Ausbildung professioneller Standards (S.343-397). Chur: Rüegger.

Buuren, S. van \& Groothuis-Oudshoorn, K. (2011). mice: multivariate imputation by chained equations in R. Journal of Statistical Software, 45(3), 1-67.

Cole, J. S. \& Gonyea, R. M. (2010). Accuracy of self-reported SAT and ACT test scores: Implications for research. Research in Higher Education, 51, 305-319. https://doi.org/10.1007/ s11162-009-9160-9

Cramer, C. (2012). Entwicklung von Professionalität in der Lehrerbildung. Empirische Befunde zu Eingangsbedingungen, Prozessmerkmalen und Ausbildungserfahrungen Lehramtsstudierender. Bad Heilbrunn: Klinkhardt.

Cramer, C. (2016a). Berufswahl Lehramt: Wer entscheidet sich warum? In M. Rothland (Hrsg.), Beruf Lehrer/Lehrerin. Ein Studienbuch (S. 261 -276). Münster: UTB.

Cramer, C. (2016b). Personale Merkmale Lehramtsstudierender als Ausgangslage der professionellen Entwicklung. Dimensionen, Befunde und deren Implikationen für die Lehrerbildung. In A. Boeger (Hrsg.), Eignung für den Lehrerberuf. Auswahl und Förderung (S. 31 -56). Wiesbaden: Springer VS. https://doi.org/10. 1007/978-3-658-10041-4_3

Deci, E. L. \& Ryan, R. M. (1993). Die Selbstbestimmungstheorie der Motivation und ihre Bedeutung für die Pädagogik. Zeitschrift für Pädagogik, 39, $223-238$.

Dörrenbacher-Ulrich, L., Biermann, A., Brünken, R. \& Perels, F. (2019). Studienwahlmotivation von Lehramtsstudierenden und
Aspekte ihrer professionellen Kompetenz. Zeitschrift für Entwicklungspsychologie und pädagogische Psychologie, 51(1), 45-61. https://doi.org/10.1026/0049-8637/a000208

Eid, M., Gollwitzer, M., \& Schmitt, M. (2017). Statistik und Forschungsmethoden. Lehrbuch. Mit Online-Material (5., korrigierte Auflage). Beltz.

Fray, L. \& Gore, J. (2018). Why people choose teaching: A scoping review of empirical studies, 2007 -2016. Teaching and Teacher Education, 75, 153 -163. https://doi.org/10.1016/j.tate.2018. 06.009

Frucot, V. G. \& Cook, G. L. (1994). Further research on the accuracy of students' self-reported grade point averages, Sat scores, and course grades. Perceptual and Motor Skills, 79, 743 - 746. https: //doi.org/10.2466/pms.1994.79.2.743

Geiser, C. (2011). Datenanalyse mit Mplus. Eine anwendungsorientierte Einführung (2. Aufl.). Wiesbaden: VS Verlag.

Hartig, J., Jude, N. \& Rauch, W. (2003). Entwicklung und Erprobung eines deutschen Big-Five-Fragebogens auf Basis des international personality item pools (IPIP40). Frankfurt am Main: Institut für Psychologie.

Heinz, M. (2015). Why choose teaching? An international review of empirical studies exploring student teachers' career motivations and levels of commitment to teaching. Educational Research and Evaluation, 21, 258-297. https://doi.org/10.1080/ 13803611.2015.1018278

Holland, J. L. (1997). Making vocational choices. A theory of vocational personalities and work environments (3rd ed.). Odessa: PAR.

Klusmann, U. (2011). Individuelle Voraussetzungen von Lehrkräften. In M. Kunter, J. Baumert, W. Blum, U. Klusmann, S. Krauss \& M. Neubrand (Hrsg.), Professionelle Kompetenz von Lehrkräften. Ergebnisse des Forschungsprogramms COACTIV (S.297-304). Münster:Waxmann.

Köller, O. (1998). Zielorientierungen und schulisches Lernen. Münster: Waxmann.

König, J., Drahmann, M. \& Rothland, M. (2018). Motivprofile von Studierenden zu Beginn der Lehrerbildung. Zeitschrift für Bildungsforschung, 8, 153 -171. https://doi.org/10.1007/s35834018-0212-0

König, J. \& Rothland, M. (2012). Motivations for choosing teaching as a career: effects on general pedagogical knowledge during initial teacher education. Asia-Pacific Journal of Teacher Education, 40, 289 - 315. https://doi.org/10.1080/1359866X.2012.700045

König, J. \& Rothland, M. (2013). Pädagogisches Wissen und berufsspezifische Motivation am Anfang der Lehrerausbildung. Zum Verhältnis von kognitiven und nicht-kognitiven Eingangsmerkmalen von Lehramtsstudierenden. Zeitschrift für Pädagogik, 59, $43-65$.

König, J., Rothland, M., Darge, K., Lünnemann, M. \& Tachtsoglou (2013). Erfassung und Struktur berufswahlrelevanter Faktoren für die Lehrerausbildung und den Lehrerberuf in Deutschland, Österreich und der Schweiz. Zeitschrift für Erziehungswissenschaft, 16, 553 - 577. https://doi.org/10.1007/s11618-013-0373-5

Kuncel, N. R., Credé, M. \& Thomas, L. L. (2005). The validity of self-reported grade point averages, class ranks, and test scores: A metaanalysis and review of the literature. Review of Educational Research, 75, 63 - 82. https://doi.org/10.3102/00346543075001063

Künsting, J. \& Lipowsky, F. (2011). Studienwahlmotivation und Persönlichkeitseigenschaften als Prädiktoren für Zufriedenheit und Strategienutzung im Lehramtsstudium. Zeitschrift für Pädagogische Psychologie, 25, 105 -114. https://doi.org/10.1024/ 1010-0652/a000038

Kunter, M. (2014). Forschung zur Lehrermotivation. In E. Terhart, H. Bennewitz \& M. Rothland (Hrsg.), Handbuch der Forschung zum Lehrerberuf (2. Aufl., S.698-711). Münster: Waxmann.

Kunter, M., Kleickmann, T., Klusmann, U. \& Richter, D. (2011). Die Entwicklung professioneller Kompetenz von Lehrkräften. In M. Kun- 
ter, J. Baumert, W. Blum, U. Klusmann, S. Krauss \& M. Neubrand (Hrsg.), Professionelle Kompetenz von Lehrkräften. Ergebnisse des Forschungsprogramms COACTIV (S. 55 - 68). Münster: Waxmann.

Lipowsky, F. (2003). Wege von der Hochschule in den Beruf. Eine empirische Studie zum beruflichen Erfolg von Lehramtsabsolventen in der Berufseinstiegsphase. Bad Heilbrunn: Klinkhardt.

Mayr, J. (2009, März). Studien-und Berufswahlmotive von Lehrpersonen. Wie sie entstehen, wie sie sich verändern und was sie bewirken. Tagungsbeitrag auf der 5. Tagung der Sektion „Empirische Bildungsforschung" der Deutschen Gesellschaft für Erziehungswissenschaft (DGfE), Landau.

Mertens, J., Sann, J., Böttger, J., Haase, J., Hannover, B. \& Braun, E. (2020). Erste Ergebnisse einer Meta-Analyse zum Einfluss nicht-kognitiver Prädiktoren auf Studienerfolg in Deutschland. Poster präsentiert auf der 15. Jahrestagung der Gesellschaft für Hochschulforschung (GfHF), Hamburg.

Möller, J. \& Trautwein, U. (2015). Selbstkonzept. In E. Wild \& J. Möller (Hg.), Pädagogische Psychologie (2. Aufl., S.177 - 199). Springer: Berlin Heidelberg.

Muthén, L. K. \& Muthén, B. O. (2015). Mplus user's guide (7th ed.). Los Angeles: Muthén \& Muthén.

Neugebauer, M. (2013). Wer entscheidet sich für ein Lehramtsstudium - und warum? Eine empirische Überprüfung der These von der Negativselektion in den Lehrerberuf. Zeitschrift für Erziehungswissenschaft, 16, 157 -184. https://doi.org/10.1007/ s11618-013-0343-y

Paulick, I. (2011). Zielorientierungen und schulisches Lernen am Grundschulübergang. Dissertation. Georg-August-Universität zu Göttingen, Göttingen.

Pohlmann, B. \& Möller, J. (2010). Fragebogen zur Erfassung der Motivation für die Wahl des Lehramtsstudiums (FEMOLA). Zeitschrift für Pädagogische Psychologie, 24, 73-84. https://doi. org/10.1024/1010-0652/a000005

Retelsdorf, J. \& Möller, J. (2012). Grundschule oder Gymnasium? Zur Motivation ein Lehramt zu studieren. Zeitschrift für Pädagogische Psychologie, 26, 5-17. https://doi.org/10.1024/10100652/a000056

Rheinberg, F. \& Vollmeyer, R. (2012). Motivation (8. Aufl.). Stuttgart: Kohlhammer.

Richardson, P.W. \& Watt, H. M. (2014). Why people choose teaching as a career. An expectancy-value approach to understanding teacher motivation. In P. W. Richardson, S. A. Karabenick \& H. M. Watt (Eds.), Teacher motivation. Theory and practice (pp.3-19). New York: Routledge.

Rothland, M. (2014). Warum entscheiden sich Studierende für den Lehrerberuf? Berufswahlmotive und berufsbezogene Überzeugungen von Lehramtsstudierenden. In E. Terhart, H. Bennewitz \& M. Rothland (Hrsg.), Handbuch der Forschung zum Lehrerberuf (2. Aufl., S.349-385). Münster: Waxmann.

Schermelleh-Engel, K., Moosbrugger, H. \& Müller, H. (2003). Evaluating the fit of structural equation models: Tests of significance and descriptive goodness-of-fit measures. Methods of Psychological Research Online, 8(2), $23-74$.

Schiefele, U., Krapp, A. \& Schreyer, I. (1993). Metaanalyse des Zusammenhangs von Interesse und schulischer Leistung. Zeitschrift für Entwicklungspsychologie und Pädagogische Psychologie, 10, $120-148$.

Schiefele, U. \& Schreyer, I. (1994). Intrinsische Lernmotivation und Lernen: ein Überblick zu Ergebnissen der Forschung. Zeitschrift für Pädagogische Psychologie, 8, 1-13.

Schiefele, U., Streblow, L., Ermgassen, U. \& Moschner, B. (2003). Lernmotivation und Lernstrategien als Bedingungen der Studienleistung. Zeitschrift für Pädagogische Psychologie, 17, 185 - 198. https://doi.org/10.1024//1010-0652.17.34.185

Schiefele, U. \& Wild, K. P. (1994). Lernstrategien im Studium. Ergebnisse zur Faktorenstruktur und Reliabilität eines neuen Frage- bogens. Zeitschrift fur Differentielle und Diagnostische Psychologie, 15, $185-200$.

Schiefele, U., Wild, K.-P. \& Winteler, A. (1995). Lernaufwand und Elaborationsstrategien als Mediatoren der Beziehung von Studieninteresse und Studienleistung. Zeitschrift für Pädagogische Psychologie, 9, $181-188$.

Schuler, H., Prochaska, M. \& Frintup, A. (2001). LMI - Leistungsmotivationsinventar. Dimensionen berufsbezogener Leistungsorientierung. Göttingen: Hogrefe.

Schunk, D. H., Meece, J. L. \& Pintrich, P. R. (2014). Motivation in education. Theory, research, and applications (4th ed.). Boston: Pearson.

Schwippert, K., Feld, I., Doll, J. \& Buchholtz, N. (2013). Vergleich motivationaler und volitionaler Bedingungen selbst eingeschätzten Studienerfolgs von Lehramtsstudierenden in zwei Studienabschnitten. In S. Blömeke, A. Bremerich-Vos, G. Kaiser, G. Nold, H. Haudeck, J.-U. Keßler et al. (Hrsg.), Professionelle Kompetenzen im Studienverlauf. Weitere Ergebnisse zur Deutsch-, Englisch- und Mathematiklehrerausbildung aus TEDS-LT (S. 231 - 249). Münster: Waxmann.

Sparfeldt, J. R., Buch, S., Rost, D. H. \& Lehmann, G. (2008). Akkuratesse selbstberichteter Zensuren. Psychologie in Erziehung und Unterricht, 55, $68-75$.

Streblow, L. \& Pohlmann, B. (2017). Studienwahlmotive, berufsbezogene Interessen und Selbstkonzepte angehender Lehrerinnen und Lehrer. In J. Retelsdorf, F. Zimmermann, A. Südkamp \& O. Köller (Hrsg.), Im Blickpunkt pädagogisch-psychologischer Forschung. Selbstbezogene Kognitionen, sprachliche Kompetenzen und Professionalisierung von Lehrkräften (S.221 - 238) Münster: Waxmann.

Trapmann, S. (2008). Mehrdimensionale Studienerfolgsprognose. Die Bedeutung kognitiver, temperamentsbedingter und motivationaler Prädiktoren für verschiedene Kriterien des Studienerfolgs. Berlin: Logos.

Trapmann, S., Hell, B., Hirn, J.-O. W. \& Schuler, H. (2007). Metaanalysis of the relationship between the big five and academic success at university. Zeitschrift Für Psychologie, 215, 132 - 151. https://doi.org/10.1027/0044-3409.215.2.132

Voss, T., Kunter, M., Seiz, J., Hoehne, V. \& Baumert, J. (2014). Die Bedeutung des pädagogisch-psychologischen Wissens von angehenden Lehrkräften für die Unterrichtsqualität. Zeitschrift für Pädagogik, 60, $184-201$.

Wolf, K., Kunina-Habenicht, O., Maurer, C. \& Kunter, M. (2018). Werden aus guten Schülerinnen und Schülern auch erfolgreiche Lehrkräfte? Zeitschrift für Pädagogische Psychologie, 32, 101-115.

\section{Historie}

Manuskript eingereicht: 19.03.2020

Manuskript nach Revision angenommen: 04.02.2021

Onlineveröffentlichung: 01.03.2021

\section{Förderung}

Open Access-Veröffentlichung ermöglicht durch die Universität Kassel.

\section{ORCID}

Caroline Theurer

(iD) https://orcid.org/0000-0002-0598-1915

\section{Caroline Theurer}

Universität Kassel

Institut für Erziehungswissenschaften

Fachgebiet Empirische Schul- und Unterrichtsforschung

Nora Platiel Str. 1

34109 Kassel

Deutschland

theurer@uni-kassel.de 